\title{
Intertexte et déviance : Lecture du Libraire
}

\author{
Jean-Jacques HAMM \\ Université Queen's
}

Le 15 octobre 1965, tard dans la soirée et après un coup de téléphone de Gérard Bessette, je me rendis à son domicile. La discussion, sur fond de nuit, où je fus à la fois interlocuteur privilégié et cible, fut intéressante, longue et vive à l'occasion. Des sujets abordés, je retiendrai celui de la place dominante que la France occupait en matière de culture et de langue, et en conséquence de la place qui était faite à un auteur québécois, forcément à l'extérieur, forcément infériorisé. La question du statut de l'écrivain et de l'œuvre, provenant tous deux du dehors d'une métropole ou d'une culture hégémonique, cette question était nouvelle pour moi à l'époque. 
Comment donc écrire, comment se définir, comment exister littérairement, alors que l'important est censé être ailleurs? ${ }^{1}$ Parmi les réponses qu'un auteur pouvait donner à ce genre de situation, j'en désignerai trois, les deux premières servant de balises par rapport à la démarche que je voudrais étudier. D'autres choix sont sans doute possibles, et la série que je propose ne se veut en aucun cas exhaustive.

J'indiquerai en premier lieu l'imitation. On pourrait faire dévier de son contexte telle formule à séduire employée par Léon dans Madame Bovary: "Cela se fait à Paris! ». Aucune raison donc de ne pas le faire à Rouen, ou à Montréal. L'imitation, étant une démarche d'adhésion au désir du père. Imiter, c'est faire allégeance ou s'affirmer inférieur. Écrire comme à Paris ne peut que dévaloriser la littérature ainsi produite. Il est certes des degrés dans l'imitation qui, lorsqu'elle n'est que partielle ou locale, peut déboucher sur la parodie, et par là même sur la liquidation d'un modèle ou d'un genre.

La seconde attitude, qui tourne le dos à la métropole, est la revendication du droit à un idiolecte local. «Au Québec, écrit François Paré, la montée du joual comme langue littéraire se produit vers 1960 [...] au moment où le milieu universitaire québécois s'attache à redéfinir profondément son allégeance au discours français dominant» (1992, p. 40). Je citerai, venant d'un autre lieu et d'un autre combat, le cas du poète Claude Vigée, dialectophone qui écrit en français, puis en 1990 produit une oeuvre bilingue, Wénderôwefîr / Le feu d'une nuit d'hiver,

\footnotetext{
${ }^{1} \mathrm{Cf}$. les pages de Gérard Tougas sur le sort des écrivains de Suisse romande, et sur celui du poète Pierre-Louis Matthey, dans Destin littéraire du Québec, 1982, p. 33-36.
} 
qui érige le dialecte en langue originelle dont le français sera la traduction.

La troisième attitude, qui est celle dont je me propose de parler, est la prise en compte d'un intertexte que l'écrivain soumet à un travail de différenciation, de déviance, travail qui est accompli sur un corpus à la fois de littérature et de langue. Le concept d'intertexte, à la fois précis et vague, sera entendu ici comme fait de structure et d'écriture, et non comme fait de lecture ou de plaisir du texte ${ }^{2}$. Dans ce cas, le rapport à l'intertexte sera vu avant tout comme agonistique. Je renvoie aux démarches et figures que Harold Bloom propose dans The Anxiety of Influence. Le corps à corps, non pas avec ce qui relève de l'air du temps mais avec ce qui appartient à des archétypes ou des formulations, nécessite la présence de signes lisibles ou repérables à même le matériau culturel. Le travail de déviance de l'écrivain permettra de juger du degré de liberté d'une oeuvre, de son degré d'innovation. Je prendrai en considération deux niveaux de structures: l'un étant macro-textuel, l'autre micro-textuel.

\section{Niveau macro-textuel}

Le Libraire met en scène d'une part un archétype narratif et d'autre part un personnage dont on peut dire qu'il participe, dans les diverses phases de son parcours, d'au moins deux types littéraires, le second déjouant, relativisant, valorisant le destin du premier.

2 Ou de «souvenir circulaire» au sens barthésien du terme. R. Barthes, Le Plaisir du texte, 1973, p. 59. 
1. L'archétype narratif est en trois temps: c'est celui d'un monde fermé, tranquille ou somnolent, dans lequel est introduit un corps étranger qui nolens volens modifie l'équilibre du lieu, et qui finit par faire l'objet d'une procédure d'expulsion, choisie ou imposée, volontaire ou subie. Ce récit archétypique s'illustrerait dans l'épisode central du mythe d'œEdipe: Thèbes sous l'emprise de la sphynge, arrivée d'un héros libérateur et conquérant, nécessité d'expulsion de celui-ci. On retrouverait ce schéma et ses variantes dans maints romans. On pourrait citer Germinal de Zola, La Peste de Camus, ou Les Démons de Dostoïevski. Bien des films sont construits sur une structure semblable. Le personnage ou l'actant de ce type de récit est soit un bouc émissaire, coupable ou innocent, soit un bruit, un parasite à éliminer. Voir à ce sujet le livre de Michel Serres, Le Parasite, et l'analyse qu'il fait, entre autres, de la trajectoire de Tartuffe.

Dans Le Libraire, Saint-Joachin est un lieu clos où l'ordre et la médiocrité règnent. La topographie même de la ville est sans complexité, étant composée de rues parallèles ou perpendiculaires. La rivière y cesse d'avoir des méandres. Le nouveau venu veut limiter ses mouvements à l'extrême: «je déteste les déplacements», dit-il (p. 108). Bouger le moins possible est l'objectif principal de Jodoin qui organise donc sa vie à partir d'une série de stations: la chambre chez Mme Bouthiller, la taverne Chez Trefflé, la salle d'attente de la gare, la librairie, elle-même divisée en quatre petites territorialités, quatre petits égoïsmes, et le capharnaüm, chambre noire, antre du savoir ou du mal. L'insistance sur un espace régulier et fixe, tracé une fois pour toutes, permet d'illustrer l'une des lois du récit, à savoir qu'un projet d'inaction ou d'action appelle son contraire. Le récit donne un démenti au projet d'inertie en 
expulsant le personnage. L'aspiration à un noli me tangere, ne me dérangez pas, aura comme conséquence que les dérangements s'accumuleront. Une loi semblable régirait la trajectoire d'un personnage comique, forçant celui-ci à atteindre le contraire de ce qu'il recherchait, ou du moins à en dévier. Ainsi, dans Don Juan de Molière, le personnage, qui se veut conquérant, est constamment en fuite. La trajectoire d'autres personnages de Molière répondrait au même schéma.

$\mathrm{Ne}$ pas faire de vagues, tel pourrait être un second objectif de Jodoin, et pourtant il ne cesse d'attirer l'attention sur lui. La réponse qu'il fait à la question de la serveuse fournit un exemple typique:

Je venais sans doute pour affaire? - D'habitude je ne réponds pas à ce genre de questions; mais, comme la serveuse s'était montrée aimable, je lui déclarai que, en un sens, on pouvait dire que je venais pour affaire, pourvu que l'on prît affaire au sens large. Elle ne sembla pas bien saisir ma réponse et hocha la tête d'un air perplexe. (p. 10)

Il est donc impossible pour Jodoin, personnage excentrique fréquentant des lieux excentriques, de garder un profil bas:

- Ah! c'est vous! s'exclama-t-il, enchanté de sa découverte. C'est vous! J'ai entendu parler de vous par des amis...

Sa réplique me prouva que, en dépit de ma vie rangée, tranquille, je jouissais à Saint-Joachin d'une solide réputation. (p. 107)

2. Les types littéraires. Le premier auquel Jodoin se rattache est celui du surnuméraire, celui qui est en trop, celui qui appartient à un grade inférieur. C'est celui dont on n'a pas besoin, celui dont l'univers n'a que faire. Si le type commence à faire son apparition au début du XIX ${ }^{\mathrm{e}}$ siècle, c'est surtout le 
$\mathrm{XX}$ siècle qui le mettra en scène : petit employé, fonctionnaire sans avenir, voyageur de commerce. Le surnuméraire n'a pas de vision stratégique de son existence, tout au plus est-il concerné par des actions tactiques, ponctuelles. Voici comment Jodoin se décrit:

je me considérai moi-même comme plutôt fini, en ce sens que je n'espérais plus atteindre à une quelconque réussite intellectuelle, sociale, pécuniaire ou simplement matrimoniale... [...] je ne me sentais plus, comme on dit, appelé à une haute destinée et [...] mes déplacements à la surface du globe me paraissaient tout à fait dépourvus de signification. (p. 154, 156)

Le surnuméraire vit dans un univers désacralisé où la répétition même est auto-référentielle. Tel vers de Jules Laforgue illustrerait fort bien la situation: «Ah! que la Vie est quotidienne...» (p. 64). Peu de référents dans Le Libraire sont des signes ou des symboles: vingt bocks de bière ne signifient que vingt bocks de bière, et de toute façon, pour citer Jodoin, « je ne suis nullement alcoolique » (p. 14). Il s'agit d'occuper ou de tuer le temps qui est, lui aussi, auto-référentiel.

Le surnuméraire est foncièrement un perdant. On trouverait dans La Nausée de Sartre l'énoncé du statut métaphysique du type: «De trop: c'était le seul rapport que je pusse établir entre ces arbres, ces grilles, ces cailloux [...] Et moi - veule, alangui, obscène, digérant, ballottant de mornes pensées - moi aussi j'étais de trop » (p. 181). La critique a donné une liste d'ascendants romanesques d'Hervé Jodoin : Meursault, Roquentin, Plume, le brave soldat Chveïk, les personnages de Kafka, de Beckett, de Sallinger (Kwaterko, 1982, p. 385, 388). Parler d'ascendants laisserait supposer une filiation, tout comme d'attribuer des influences à la création. Je renvoie à ce 
qu'en disait Roland Barthes dans «Je ne crois pas aux influences $»^{3}$. À ce qu'il me semble, il y a circulation d'une matière culturelle et langagière, processus de saisie, d'appropriation, puis de distanciation, de séparation et de liquidation. Que valent quelques-uns des rapprochements proposés? Kafka: au centre de son univers se trouvent les questions de la culpabilité, de la grâce, d'un combat contre des ennemis cachés ou sournois. L'univers de Kafka propose une recherche labyrinthique d'un absolu conçu sous une forme négative. Au contraire, à Saint-Joachin, le héros sait à quoi s'en tenir : une action amène une réaction. Jodoin n'a rien non plus de l'intellectualité de Roquentin, qui finit par proposer une sortie du néant à travers la littérature, le roman, c'est-à-dire le retour à un monde qu'il n'avait cessé de dénoncer. Quant à l'Étranger ${ }^{4}$, il s'agit certes d'un même type de récit, on trouve certes une égale indifférence chez les deux personnages quant au monde qui les entoure, mais Meursault est une figure tragique, qui est forcé de payer dans sa personne l'enjeu d'un combat qu'il n'a pas choisi, et pour lequel il ne s'est pas engagé. Meursault a un destin; Jodoin a une aventure. Le monde de Camus et celui de Kafka mettent en jeu des forces autres que sociales; ce n'est pas le cas de celui de Bessette. On a cité Plume de Michaux, mais celui-ci est dans le fantastique du langage, dans la capacité d'une osmose entre le signe et son référent. De tous les exemples suggérés, la proximité la plus intéressante d'un point de vue heuristique me semble être celle de Jodoin et du brave soldat Chvéik. Ce sont tous deux des fauteurs de

\footnotetext{
3 R. Barthes préfère le concept de circulation à celui d'influence.

${ }^{4}$ Pour les ressemblances / différences, cf. J. Allard, 1970b, p. 19; A. Brochu, 1974, p. 101-102; G. Marcotte, 1976, p. 44-49.
} 
troubles par leur langage, par leur manière de s'exprimer, par des figures qui déroutent l'interlocuteur:

- Jésus-Marie, m’en v'là d'une nouvelle! s'écria Chvéïk. Et où estce que ça lui est arrivé à l'archiduc, voyons?

- À Saraiévo. Des coups de revolver. Il y était allé avec son archiduchesse en auto.

- Ça, par exemple! Ben oui, en auto... Vous voyez ce qu'c'est, M'ame Muller, on s'achète une auto et on ne pense pas à la fin... Un déplacement, ça peut toujours mal finir... (Hašek, 1964, p. 2)

Personnage de surnuméraire, excentrique et cynique, selon la réputation que ses confrères lui ont donnée, Jodoin peut se rattacher à un autre type, gagnant cette fois-ci. On peut qualifier Jodoin d'anarchiste. Le concept, tel que je l'applique ici, demande explication. Je passerai par un texte-parenthèse pour illustrer ma pensée. Il s'agit d'un passage du poète portugais Fernando Pessoa, extrait du Banquier anarchiste, pamphlet écrit en 1922 et traduit en 1988. Voici comment le personnage situe, théorise et justifie son action:

L'anarchiste idiot qui jette des bombes et tire dans la foule, sait bien qu'il va tuer alors que ses doctrines excluent la peine de mort. [...] Son acte est stupide, dans la mesure où sa démarche est erronée et même nocive, en tant que démarche anarchiste [...]. [L]a voie que je suivais était la bonne, et je me suis légitimement servi, en anarchiste convaincu, de tous les moyens pour m'enrichir. Aujourd'hui, j'ai réalisé [...] le rêve bien circonscrit d'un anarchiste pratique et lucide. Je suis libre. [...] Ma devise d'anarchiste était la liberté [...]. J'ai rendu libre un homme: je me suis rendu libre. La voie que j'ai empruntée, la seule véritablement anarchiste [...] ne m'a pas permis d'en libérer davantage. [...] En moi - oui, en moi, banquier, grand commerçant, accapareur si vous y tenez - , en moi, la théorie et la pratique de l'anarchisme se trouvent enfin exactes l'une et l'autre. (p. 83-86, 10) 
Tout comme le banquier anarchiste de Pessoa, mais sans réflexion théorique aucune, Jodoin obtient la tranquillité et la liberté souhaitées en exploitant à son profit le système de la circulation en sous-main de bien culturels, en le faisant servir à son égoïsme personnel. L'idéal de Jodoin étant le non-agir, il y parvient. Point n'est besoin pour lui de s'occuper des autres: « le chômeur pouvait toujours se démerder tout seul » (p. 22). Jodoin lui aussi a libéré qui il pouvait libérer.

L'histoire de Jodoin, par un indice, reflète le décor dans lequel il vit, alors qu'il est surtout un personnage qui apparaît anomique par rapport au monde. Pourquoi la ville s'appelle-telle Saint-Joachin? Il est fait allusion dans le roman à deux personnages dont la performance sexuelle laisse à désirer : Jodoin («Je n'ai plus l'allant d'autrefois - qui n'a d'ailleurs jamais été bien spectaculaire» (p. 100)) et le gendre de Mme Boutiller («Mme Bouthiller soupçonne son gendre d'impuissance. Au cours de la guerre il a été blessé - un éclat d'obus - et il n'y a jamais eu moyen de savoir où. Alors, n'est-ce pas, on pouvait tout supposer» (p. 66)). Selon Jacques de Voragine, Joachim aurait été repoussé du temple, parce qu'il était infécond. Dieu interviendra par la suite pour lui (re)donner sa virilité (1998, p. 495).

Personnage laconique, voire pervers lorsqu'il fume des cigarillos pour faire tousser les autres (p. 152), ayant une éducation collégiale mais sans emploi, ex-pédagogue qui ne lit plus de livres, Jodoin refuse de donner des conseils littéraires, n'aime plus les discussions sur des idées générales. Les livres, avec plus d'honnêteté que Léon Chicoine, ne sont pour lui qu'une valeur d'échange concrète: ils brûlent mieux que le charbon. S'il écrit, ce n'est pas par engagement, pour porter 
témoignage, pour faire un roman ou une œuvre - il n'a pas de destinataire - s'il écrit c'est, selon un motif qui se répète, pour «tuer le temps» (p. 11, 12, 15). Écrire tient donc du divertissement, au sens où l'entendrait Pascal; écrire c'est échapper, c'est donner du plein à ce qui est vide. S'il était pourtant un engagement de Jodoin, voire un combat, c'est au niveau micro-textuel qu'il se repèrerait, dans le rapport du personnage à la langue, aux possibilités qu'offrent l'agencement du discours et les figures qui le déstabilisent.

\section{Niveau micro-textuel}

1. Double orientation de la langue et du style. Jodoin écrivant adhère à une conception du style où l'on peut distinguer deux orientations. La première relève d'un code que l'on pourrait appeler, faute de mieux, le bien écrire en français, et ceci avec économie. On noterait l'abondance de propositions elliptiques, exemples particuliers d'une tendance stylistique plus générale : la parataxe, c'est-à-dire l'alignement de phrases sans liaisons conjonctives. Relevons la mise à distance d'expressions convenues par des commentaires du type: "comme on dit» (p. 116), «si l'on peut dire» (p. 121). Citons la mise entre guillemets d'expressions qui appartiennent à l'idiome québécois, comme "veuve à l'herbe » (p. 58). On noterait également le recours réitéré à des temps du passé du subjonctif:

Non qu'elle eût renoncé à obtenir des renseignements... (p. 63); Il eût suffi que je lève la tête [...] pour qu'elles m'assaillissent de questions (p. 111);

bien que je fusse son subordonné... (p. 112);

elle exprima la crainte que je ne me rendisse pas pleinement compte... (p. 131). 
Jodoin respecte la concordance des temps. Son journal, à son image, n'a rien de débridé. C'est une mise en ordre rigoureuse. Le narrateur écrit donc avec précision et netteté, comme en un reflet ordonné d'une vie qui se veut ordonnée.

Cette première orientation du style est minée par le recours à divers registres et niveaux de langues, et qui introduit dans le texte un élément moins formel. Voici des phrases en langage parlé :

ç’a été de me chercher une chambre (p. 7);

Ça n'avait pas d'importance (p. 7);

Ça n'a d'ailleurs aucune importance, pour moi (p. 9);

N'empêche que c'est moi qui... (p. 15);

ça ne valait pas la peine de s'éreinter (p. 33);

Ça on n'y peut rien (p. 40);

La seule chose que je peux pas vous permettre... (p. 108).

Le père Manseau, lorsqu'il parle enfin, introduit le québécois dans le récit: "Moué, c'est pas de mes affaires. Mais vous êtes nouveau icitte. [...] Les ficelles, c'est eux autres qui les ont » (p. 126). Par ailleurs, le langage est par moments familier, vulgaire, injurieux :

[...] vieilles filles constipées, ne me laissaient pas tripoter les étudiantes (p. 23);

la binette de Nault (p. 24);

ces zigotos-là (p. 72);

Il m'a alors reluqué d'un drôle d'air (p. 114);

J'ai dû roupiller un peu (p. 94);

le train s'est ramené (p. 106);

Je m'en fous (p. 109);

je me suis mis à la peloter (p. 97);

il pouvait toujours, comme on dit, se gratter le cul (p. 70);

bon à rien, un sans cœur, un flanc mou, une crapule de la pire espèce et un saligaud comme il ne s'en fabrique plus (p. 65, 92). 
Quelquefois, au contraire, un vocabulaire abstrait qualifie de manière précise des façons de parler: «amphigourique, circonlocutions, objurgations». Un mot est recherché ou «littéraire»: «lupanar» mis pour bordel, une «voix strangulée»; ou spécialisé, comme «face de prognathe » (p. 165), des « [y] eux fibrillés de rouge » (p. 147). « Forniquer» appartient au langage de la religion. On trouve un mélange de registres. Pour décrire un bourrelet on $\mathrm{a}:$ « une espèce de pneu dans la région de l'épigastre» (p. 102); ou une citation littéraire: "elle était, comme on dit, dans le simple appareil » (p. 101). On note donc la déstabilisation d'un style uniforme par un ensemble de déviances lexicales.

2. Niveau discursif. Françoise Iqbal, dans son article sur la préciosité, estimait à propos de Jodoin «que les structures mentales de son être sont parallèles à celles de la collectivité à laquelle il appartient » (1976, p. 347). L'affirmation est à la fois exacte et problématique. Des enjeux importants apparaissent au niveau discursif, qui vont certes dans le sens d'une imitation des manières de penser de la collectivité, mais qui introduisent également un ferment pour défaire le conformisme de celle-ci.

Par exemple: la méfiance étant nécessaire, la mauvaise foi étant générale, Jodoin utilise une tactique de mauvaise foi dont il a parfaitement conscience : «Au fond, je ne sais pourquoi je joue ainsi à cache-cache avec elle. Il y a bien des renseignements que je pourrais lui donner sans me faire violence. Peu importe. J'ai commencé ainsi. Autant vaut continuer » (p. 65).

Commode est alors le recours à l'alternative qui, en énonçant deux possibilités, laisse l'interlocuteur en suspens. Je renvoie à la discussion entre Mme Boutiller et Jodoin sur 
l'architecture de l'église, sur la langue parlée à Saint-Joachin, ou encore sur la taille de la ville: «- Je répondis que tout était relatif: j'avais vu, certes, des agglomérations plus considérables que Saint-Joachin, mais j'en avais vu aussi de plus petites » (p. 64).

Une société médiocre, qui fonctionne sur la peur, qui réprime la pensée qu'elle craint, qui fait tout pour empêcher la circulation des idées, qui peut à volonté exclure, ne mérite pas qu'on s'exprime clairement aussi longtemps qu'on est en position d'être anéanti. Dans une telle société, le discours sibyllin est de bonne guerre. Les scènes de Jodoin avec Chicoine, puis avec le curé sont des victoires tactiques, alors que l'adversaire pense avoir obtenu d'une part l'adhésion, d'autre part la confirmation de la bêtise du vendeur.

3. La syllepse. Mais Jodoin, par ailleurs, est un anarchiste et un être subversif. L'une des figures de rhétorique qu'il pratique le dirait assez, à savoir: la syllepse. Celle-ci consiste à utiliser le même mot dans des sens différents, l'un étant généralement le sens propre et l'autre étant le sens figuré. Il n'est d'ailleurs pas nécessaire qu'il y ait répétition explicite. Il peut arriver de plus que le mot soit absent, mais qu'il y ait emploi d'une notion dans deux sens différents ${ }^{5}$. La réponse de Jodoin à la serveuse, le jeu sur le mot "affaire», est une syllepse. À la question de Nault pour savoir si Jodoin aimait toujours les livres, il répond qu'« il [lui] arrivait de [s]'en servir» (p. 24). Le contexte explicite les sens à donner à ce verbe. L'expression «personnes sérieuses » (cf. p. 70, 117), qui

5 Sur l'emploi de la syllepse grammaticale, cf. A. Piette, Gérard Bessette. L'Incubation et ses figures, 1983, p. 71-73. 
demande explication, tient de la syllepse, de même «livres différents » (p. 123), et l'utilisation du mot «mœurs » (p. 124). Jodoin répète à plusieurs reprises qu'il s'agit pour lui de « tuer le temps » (p. 11, 12, 15). C'est à la fois le passer et l'effacer en le remplissant. L'ironie du roman, qui est décapante, fonctionne d'ailleurs par passage du sens d'un mot à l'autre, par passage d'une catégorie logique à une autre. Tout comme Chvéik qui ramène l'assassinat de l'archiduc au danger d'avoir une voiture, Jodoin ramène la question des conséquences de la blessure du gendre de sa logeuse à celle de l'imprévisibilité des éclats d'obus (p. 66). De même, la réponse à Chicoine, à propos de la visite du curé, joue sur le sens littéral et figuré du mot « comprendre» (p. 114).

4. La dialectique. Autre pratique de Jodoin: la subversion par la dialectique. Qu'il écrive dans son journal ou qu'il réponde à un interlocuteur, Jodoin fait subir à la vérité un processus dialectique. Voici comment il analyse ses habitudes Chez Trefflé:

En apparence, nulle pression extérieure ne s'exerce sur moi. [Thèse]

Toutefois, il faut tenir compte des circonstances. [Antithèse] De plus, il ne faut pas oublier que je passe en moyenne sept heures par jour Chez Trefflé.

Cela crée tout de même une certaine obligation... (p. 15-16). [Synthèse]

On peut relever le mouvement dialectique par lequel il se demande si Mme Bouthiller est du « genre collant» (p. 56-57), par lequel il décide s'il est satisfait (p. 59). Il conviendrait de citer la réponse au curé à propos de l'Essai sur les mœurs :

[...] je ne pensais pas qu'il vint de notre librairie, attendu que nos livres portaient le nom de notre établissement sur un collant à l'intérieur de la couverture [...]. 
J'ajoutai que, naturellement, cette étiquette aurait pu se décoller [...].

Toutefois [...] j'inclinais très fortement à penser que le volume ne provenait pas de chez nous. (p. 81-82)

On trouve également une démarche dialectique dans les discussions avec Chicoine :

- Ainsi, vous avez l'audace de l'avouer!

Je lui soulignai qu'il ne s'agissait pas [...] d'un aveu mais que, même si c'en était un, je ne voyais pas en quoi [...]. (p. 116; voir aussi p. 120)

Lorsque la dialectique n'est pas régie au départ par des présupposés idéologiques qui en falsifient le mouvement, elle peut être l'ennemie des totalitarismes, des régimes à pensée unique. Le problème pour Jodoin est qu'il est le seul à pratiquer la dialectique. Il ne peut donc pas parler pour communiquer ou convaincre, mais uniquement pour se situer ailleurs, hors centre. Il s'agit de faire travailler le discours contre l'ordre établi, alors même qu'on est réduit à ne pas être entendu. La dialectique devient ainsi une simple hygiène mentale, moyen solitaire de s'assurer qu'on pense encore. La lucidité, et Jodoin est lucide, consiste à savoir où et comment se battre, c'est-àdire à savoir où et comment ne pas se battre. On pourrait reprendre, pour l'appliquer au narrateur, un concept de Nietzsche, celui de la taupe qui taraude la base, "ceux qui forent, qui sapent, qui minent » (1970, p. 13-14).

Dans Le Libraire, Gérard Bessette se bat sur deux fronts. Il est d'abord face à une culture et à une langue dominantes. Il est ensuite face à la dictature obscurantiste de l'Église catholique. Reprenant l'archétype de l'étranger, reçu puis sacrifié, le roman le modifie en triomphe du surnuméraire, personnage qu'on peut réécrire en anarchiste. Le roman illustre le conflit pérenne 
entre la liberté de l'individu et l'oppression sociale. Il montre qu'il n'y a de salut qu'individuel dans une communauté somnolente ou lâche. La solidarité, lorsqu'elle existe, est de personne à personne et elle est tacite, le langage étant piégé. Voir les rapports entre Jodoin et le père Manseau.

Le schéma de ce roman dévie donc de bien des romans où le surnuméraire est écrasé par l'ordre régnant. Jodoin sort vainqueur de Saint-Joachin. S'exprimant dans la langue dominante, Jodoin la respecte d'une part et la déstabilise de l'autre. Il n'imite pas le français, il ne rompt pas avec lui, il le carnavalise, le rendant hybride. Complice en apparence de l'ordre des choses, Jodoin mine les certitudes par une rhétorique de l'ambiguïté. Le personnage est un ferment, un grain semé pour on ne sait encore quel destin, quelle incertaine liberté.

Finalement, au-delà d'une confrontation sur fond de nuit, il convenait de reconnaître en ce roman la valeur heuristique d'un corps à corps avec un intertexte et une langue, le travail de déviance qui en est résulté. Une création, qui passe par ce processus-là, qui est donc invention d'une forme, mérite qu'on continue à s'intéresser à elle.

\section{Bibliographie}

AllARD, Jacques (1970a), «Le Libraire de Gérard Bessette ou comment la parole vient au pays ", Voix et images au pays, no 1, p. 51-63.

— (1970b), Le Libraire, Montréal, Éd. du Renouveau pédagogique. 
BARTHES, Roland. 1981, "Je ne crois pas aux influences", dans Le grain de la voix, Paris, Seuil, coll. « Essais », p. 29-35.

Barthes, Roland. 1973, Le Plaisir du texte, Paris, Seuil.

BESSETTE, Gérard. 1960, Le Libraire, Paris, Julliard.

BLOOM, Harold. 1975, The Anxiety of Influence, London, New York, Oxford University Press.

BRochu, André. 1974, L'Instance critique 1961-1973, Montréal, Leméac.

HAŠEK, Jaroslav. 1964, Le Brave Soldat Chvéïk, Paris, Club français du livre.

IQBAL, Françoise. 1976, « Précieux et préciosité chez Bessette: demimesure et démesure », Voix et images, vol. 1, n 3, p. 338-364.

KWATERKO, Józef. 1982, «Le sarcasme dissident: une lecture politique du Libraire »,Voix et images, vol. VII, n 2, p. 385-393.

LAFORGUE, Jules. 1993, Que la vie est quotidienne, Paris, La Différence.

MARCOTTE, Gilles. 1976, Le Roman à l'imparfait, Montréal, La Presse.

NIETZSCHE, Friedrich. 1970 [1881], Aurore, Paris, Gallimard, coll. «Idées ».

PARÉ, François. 1992, Les Littératures de l'exiguïté, Hearst, le Nordir.

PESSOA, Fernando. 1988, Le Banquier anarchiste, Paris, La Différence.

PIETTE, Alain. 1983, Gérard Bessette. L'Incubation et ses figures, Montréal, Presses de l'Université de Montréal.

SARTRE, Jean-Paul. 1966 [1938], La Nausée, Paris, Livre de poche.

SERrES, Michel. 1980, Le Parasite, Paris, Grasset.

TougAS, Gérard. 1982, Destin littéraire du Québec, Montréal, Québec / Amérique.

VIGÉE, Claude. 1990, Wénderôwefîr/Le feu d'une nuit d'hiver, Strasbourg. 
Voragine, Jacques de. 1998, La Légende dorée, Paris, Seuil, coll. « Points ».

\title{
Résumé
}

Cet article explore l'un des choix auxquels fait face un écrivain confronté à une tradition littéraire dominante: imitation, promotion d'idiolecte régional, travail de différentiation et de déviance par rapport à un intertexte de littérature et de langue. C'est cette troisième voie que l'on peut observer dans Le Libraire de Gérard Bessette, à la fois au niveau macrotextuel (archétype narratif, type littéraire) et au niveau microtextuel (langue et style, niveau discursif, rhétorique, dialectique). C'est par un travail de déviance de désécriture, d'invention d'une forme que l'œuvre acquiert un statut qui la libère des contraintes d'une tradition et d'une autre culture.

\begin{abstract}
This article explores one of the choices a writer is faced with when confronted with a dominant tradition: imitation, promotion of a regional language, rewriting, through transformation and modification, of a literary intertext and its language. It is this third option that one sees at work in Gérard Besseette's Le Libraire, on both the macro-textual level (narrative archetypes, characters) and the micro-textual level (language and style, discourse, rhetoric, dialectics). It is through these transformations and deviances that the work states its independence from the constraining models pertaining to another tradition and culture.
\end{abstract}

